\title{
Femoral arteriovenous fistula associated with surgery of proximal femoral fracture: a systematic review of the literature and case presentation
}

\author{
Jun Seok Kim ${ }^{1}$, Song Am Lee ${ }^{1}$, Hyun Keun Chee ${ }^{1}$, Jae Joon Hwang ${ }^{1}$, Hye Young Kim², Jin Yong Kim , \\ Seung Myung Choi ${ }^{4}$, Yo Han $\mathrm{Kim}^{5}$, Woo Surng Lee ${ }^{5}$ \\ ${ }^{1}$ Department of Thoracic and Cardiovascular Surgery, School of Medicine, Konkuk University, Konkuk University Seoul Hospital, Seoul, Republic \\ of Korea; ${ }^{2}$ Department of Anesthesia and Pain Medicine, ${ }^{3}$ Department of Emergency Medicine, ${ }^{4}$ Department of Orthopedic Surgery, ${ }^{5}$ Department \\ of Thoracic and Cardiovascular Surgery, School of Medicine, Konkuk University, Konkuk University Chungju Hospital, Chungju-si, Chungbuk, \\ Republic of Korea \\ Contributions: (I) Conception and design: All authors; (II) Administrative support: JS Kim, WS Lee; (III) Provision of study materials or patients: JS \\ Kim, HY Kim, WS Lee; (IV) Collection and assembly of data: JS Kim, WS Lee, SA Lee; (V) Data analysis and interpretation: JS Kim, HY Kim, WS \\ Lee, SA Lee, JY Kim, SM Choi, YH Kim, HK Chee; (VI) Manuscript writing: All authors; (VII) Final approval of manuscript: All authors. \\ Correspondence to: Woo Surng Lee, MD, PhD. Professor, Department of Thoracic and Cardiovascular Surgery, School of Medicine, Konkuk \\ University, Konkuk University Chungju Hospital, 82, Gugwon-daero, Chungju-si, Chungbuk, Republic of Korea. Email: timesgoby@naver.com.
}

Background: Proximal femoral fracture (PFF), such as intertrochanteric femoral fracture or femur neck fracture, and its management are crucial issues to surgeons. PFF has been dramatically is becoming exponentially prevalent, and it is at high risk of complication and mortality because it is frequently associated with serious trauma and advanced age, especially in patients treated with anticoagulants or antiplatelet agents. Surgical management is essential for the treatment of PFF. Unfortunately, current surgical procedures have been related to accompanied by vascular complications, including laceration, hemorrhage, thrombosis, embolism, intimal flap tear and pseudoaneurysm. Furthermore, these vascular injuries following surgical management of PFF are potentially limb- and life-threatening. Of the complications after operation of $\mathrm{PFF}$, femoral arteriovenous fistula (AVF) is rare, but remains a challenging problem because it is frequently associated with significantly high mortality and morbidity and is very difficult to treat.

Methods: A systematic literature review was conducted using the PRISMA guidelines with no language restriction. We searched scientific publications via PubMed, Embase, Cochrane central register of controlled trial, Google Scholar, the KoreaMed and the Research Information Sharing Service database. The goal of this study was to report on the incidence, clinical presentation, diagnosis, treatment, associated complications, morbidity and mortality of femoral AVF caused by PFF and to draw special attention to its prevention and management.

Results: A total of 7 case reports on femoral AVF associated with operation of PFF were identified, and one our case was added to the systematic analysis. Of the 8 cases, 4 were male and 4 were female under the age of $67.87 \pm 18.44$; $6(75.0 \%)$ survived without any events, $1(12.5 \%)$ survived with a sequela of peroneal nerve impairment, and 1 (12.5\%) died of multi-organ failure and hypovolemia.

Conclusions: The incidence of femoral AVF associated with PFF is extremely low, though it appears to increase with the rising frequency of PFF. With a very few exceptions, complications following internal fixation are potentially limb- and life-threatening. There is still no definite consensus on the standardized diagnostic or therapeutic modalities. Therefore, surgeons should keep in mind that this serious complication requires early diagnosis and prompt treatment, which should not be underestimated. Femoral AVF following operation of PFF should be meticulously managed, because untreated fistulae result in serious unexpected complications including renin-mediated hypertension, high-output heart failure and venous and/or arterial insufficiency. Surgical treatment is still the gold standard for such cases, but in limited cases endovascular procedures using embolization and closure device can be a good treatment option. 
Keywords: Arteriovenous fistula (AVF); vascular fistula; hip fractures; femoral neck fractures; inter-trochanteric femoral fractures

Submitted Dec 07, 2019. Accepted for publication Feb 14, 2020.

doi: $10.21037 /$ atm.2020.03.08

View this article at: http://dx.doi.org/10.21037/atm.2020.03.08

\section{Introduction}

Proximal femoral fracture (PFF), including femoral neck, intertrochanteric or subtrochanteric fracture, is significantly associated with advanced age and osteoporosis. This disease entity has been prevalent due to the expansion of elderly populations and is expected to dramatically rise during the next decade. PFF has exponentially increased and it is at high risk of complication and mortality because it is frequently associated with serious trauma, unexpected complications, significant delayed sequelae, poor general condition at advanced ages and underlying disease. Therefore, the number of patients with PFF requiring hip surgical procedures has been increasing, and hip surgery procedures, including open reduction, internal fixation, retraction, instrumentation or exothermic reaction, are mandatory in most cases. The incidence of iatrogenic arterial trauma associated with orthopedic surgery has been rising in recent years. Orthopedic surgery has been related to various postoperative complications, one of which is vascular injury, such as laceration, hemorrhage, thrombosis, embolism, intimal flap tear or pseudoaneurysm. Femoral vessel injury is extremely rare in PFF cases, with an incidence of $<0.21 \%(1-3)$. Unfortunately, vascular injury as a complication is frequently overlooked, and it is potentially limb- and life-threatening due to delayed diagnosis and treatment. Of the complications associated with operation of PFF, femoral arteriovenous fistula $(\mathrm{AVF})$ is rare, but remains a challenging problem because it frequently has high mortality and morbidity and it is very difficult to treat $(4,5)$. This report describes a case of femoral $\mathrm{AVF}$ in a patient undergoing internal fixation of FPP and discusses its clinical presentation, diagnosis and treatment with a detailed systematic review.

\section{Methods}

\section{Literature review}

This systematic review was conducted in accordance with the Preferred Reporting Items for Systematic Reviews and Meta-Analyses (PRISMA) guidelines (6). Data were collected according to a standardized protocol; the objectives and exclusion/inclusion criteria were specified in detail. We systematically scrutinized the issues related using the following databases: the PubMed, Embase, Cochrane Library (Cochrane Central Register of Controlled Trial), Google Scholar, Springer, Science Direct, KoreaMed, OvidSP, Dialnet, J-Stage, and Scielo and the Research Information Sharing Service database. Scientific publications were searched for the following keywords: "Femoral Fractures"[Mesh] OR "Hip Fractures"[TW] OR "Hip Fractures"[TW] OR "Femoral Neck Fractures"[TW] AND/OR "Inter-Trochanteric Femoral Fractures"[TIAB] AND/OR "Sub-Trochanteric Femoral Fractures"[TIAB] AND/OR "Fracture Fixation"[TIAB] AND/OR "Fracture Fixation"[TIAB] AND/OR "Orthopedic Surgery"[TIAB] AND/OR "Vascular Complications in Orthopedic Surgery"[TIAB] AND/OR "Hip Injuries"[TIAB] AND/OR "Vascular Postoperative Complication" [TIAB] OR "Arteriovenous Fistula (AVF)"[Mesh] OR "Vascular Injury"[TW] OR "Vascular System Injuries"[TW] OR "Femoral Vessel Injury" [TW] OR "Vascular Fistula" [TW] OR "Femoral Artery Lesion"[TIAB] OR "Laceration"[TIAB] AND/OR "Hemorrhage"[TIAB] AND/OR "Thrombosis"[TIAB] AND/OR "Intimal Flap Tear"[TIAB] AND/OR "Pseudoaneurysm"[TIAB]. Two investigators (Woo Surng Lee and Jun Seok Kim) independently evaluated the eligibility of relevant abstracts and full articles, and all pertinent references of the articles written in any kind of language were also reviewed. No date restriction was applied to the journal evaluation. In cases of disagreement between the 2 investigators, further discussions were made with a third investigator (Song Am Lee). The initial data was evaluated on February 01, 2019 and a final journal search was performed on November 30, 2019. Only 7 case reports were found, without any randomized control clinical trials or comparative studies (7). The information on the history of hip operation, time to presentation, surgical treatment, location of injured vessels and mechanism of injury was analyzed. The exclusion criteria were as follows: (I) vascular lesion caused by other 
type of femur fracture, such as femur shaft fracture or distal femur fracture; (II) gunshot wound to the femur; (III) relatively common type of surgical complications, such as false aneurysm, hemorrhage, ischemia, ischemia plus hemorrhage, thromboembolic lesion, thrombosis, embolism, laceration, intimal flap tear or pseudoaneurysm; (IV) underlying vascular lesions, such as abdominal aortic aneurysm or pseudoaneurysm; (V) unusual medical conditions, such as carcinoma, malignancy or metastatic lesion; and (VI) venous complications by pelvic varix or rupture. This study was approved by the Institutional Ethics Committee/Review Board of Konkuk University Chungju Hospital, which waived informed consent due to its retrospective nature of study (IRB approval No. KUCH 2019-10-032).

\section{Statistical analysis}

We obtained 7 case reports and 1 review article. The 7 cases were analyzed using descriptive statistics. As all the of the collected data were forms of case report and case series, except 1 meta-analysis. For these reasons, universal statistical analysis was not possible, only descriptive statistic evaluations were presented in this study.

\section{Presentation case}

A 75-year-old man presented to the Emergency Department after a motorcycle traffic accident struck by a 25-ton dump truck. He had no remarkable history of specific disease or operation except for hypertension successfully treated with calcium-channel blockers at a regional hospital for 25 years. He was a farmer his whole life. At the time of the traffic accident, he was wearing a crash helmet. At presentation, he showed a loss of consciousness, and his Glasgow coma scale (GCS) score was 12 points: eye response, 3; verbal response, 4; and motor response, 5. His blood pressure was $110 / 75 \mathrm{mmHg}$, pulse rate was 98 beats/min, respiratory rate was $22 / \mathrm{min}$, body temperature was $36.8{ }^{\circ} \mathrm{C}$, and oxygen saturation measured using a pulse oximeter was $96 \%$. Physical examination revealed a deep bruise in his face, mild nasal bleeding, severe pain and a deformity in the left hip area, with no tenderness or rebound tenderness in the chest, abdomen or pelvis. Extended focused assessment with sonography for trauma (FAST) was performed in the thorax and abdomen, which revealed neither intra-abdominal fluid collections nor thorax lesions. Motor disturbance was observed in the left lower extremity, but distal upper and lower limb pulses and sensations were normal. After the initial resuscitation and management, his blood pressure became $125 / 85 \mathrm{mmHg}$, pulse rate became 80 beats/min, respiratory rate became $20 /$ min, body temperature became $36.8^{\circ} \mathrm{C}$, and oxygen saturation measured using a pulse oximeter became $98 \%$. Laboratory tests revealed WBC, 10,200/uL and hemoglobin, $14.5 \mathrm{~g} / \mathrm{dL}$. Chest plain radiographs as well as brain, chest, abdomen and pelvis computed tomography (CT) scans with intravenous contrast media revealed the following findings: compound comminuted depressed skull fracture (FCCD) in the right frontal-temporal area, right frontal sinus fracture, traumatic subdural hematoma $(\mathrm{SDH}) /$ intracranial hemorrhage (ICH) in the right frontaltemporal area, basal skull fracture, and left femoral intertrochanteric fracture. An emergency operation-ICH and SDH removal, frontal sinus repair and canalization, and repair of FCCD-was performed by the neurosurgeons. After surgical treatment, his mental state became alert, and he was closely managed in the intensive care unit (ICU) for 4 days. On the fifth day in the ICU, follow-up laboratory tests and brain CT scans confirmed complete improvements in the clinical condition. He was transferred to the general ward, resumed a normal diet, and showed improvement in daily life activities. On the 17 th hospital day, he underwent closed reduction and internal fixation with proximal femur intramedullary nailing for the left femur intertrochanteric fracture by the orthopedic surgeons under general anesthesia, and his clinical course progressed uneventfully after operation.

On the 7 th postoperative day, a gradually increasing swelling developed in the left lower extremity, but it was neglected. Two days later, the swelling worsened, and a left leg pain developed. Physical examination showed left lower extremity swelling, slight elevation in skin temperature, venous dilatation in the left leg, increased diameter of the left lower extremity, and a palpable thrill on the left femoral region. Duplex Doppler examination revealed femoral arterial flow into femoral venous structures through arterial venous fistulas. Contrast enhanced CT angiography exhibited (I) a suspicious fistula between the right common, superficial or deep femoral artery and its accompanying vein, (II) enhanced femoral vein, iliac vein and inferior vena cava at the arterial CT phase, and (III) dilated right femoral and iliac veins (Figures 1-3). Transthoracic echocardiography (TTE) demonstrated preserved ejection fraction and significant bi-atrial enlargement. A cardiologist attempted external compression and endovascular repair of AVF, but 

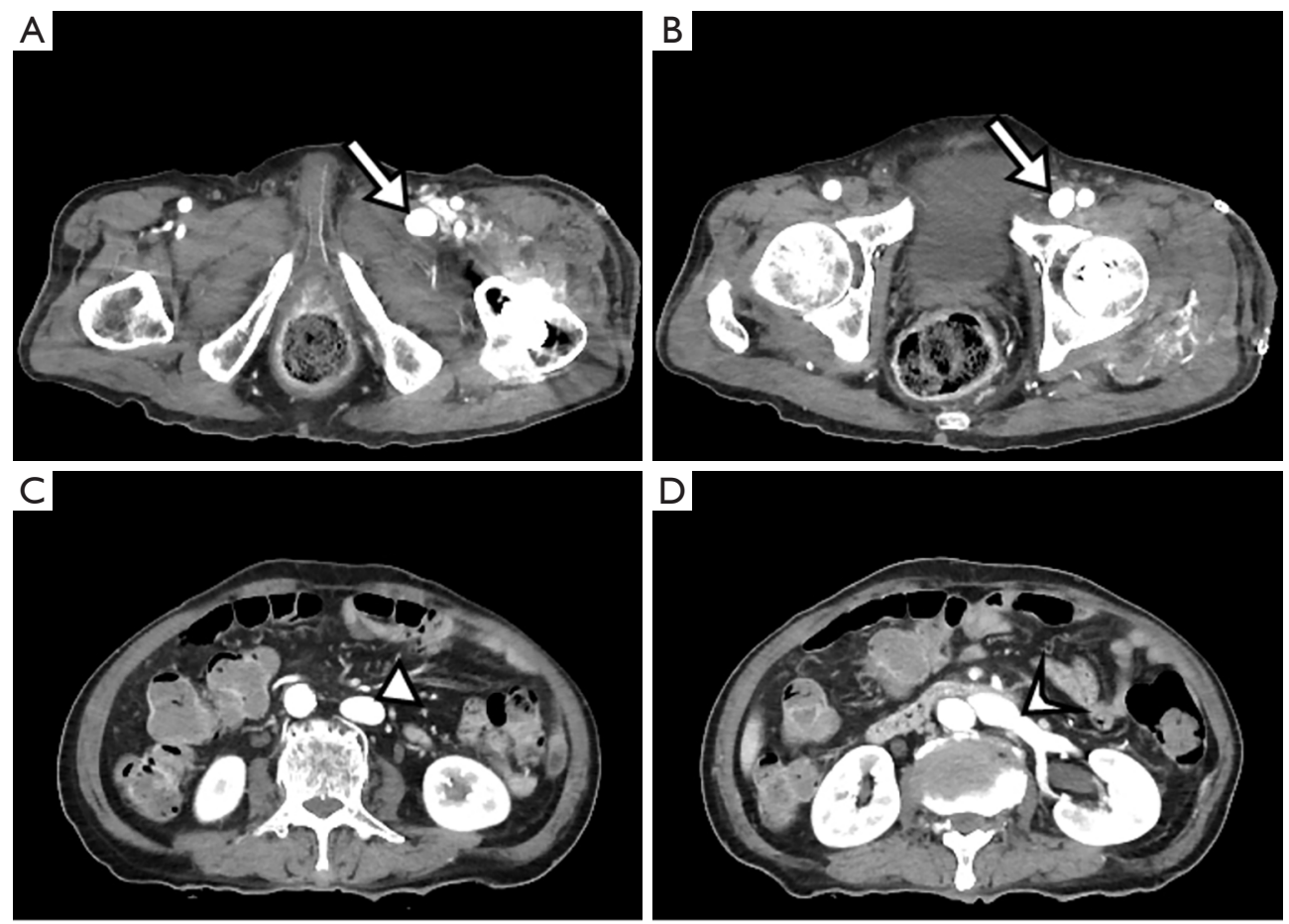

Figure 1 Preoperative transverse enhanced angiographic CT scans show abnormally enhanced veins at the arterial phase, resulting from a left-sided femoral arteriovenous fistula associated with proximal femoral fracture. White arrows, arrow heads and wedges indicate enhanced left femoral veins (A,B), inferior vena cava (C) and renal vein (D) at the arterial phase. Note the enhanced left gluteal veins (B).
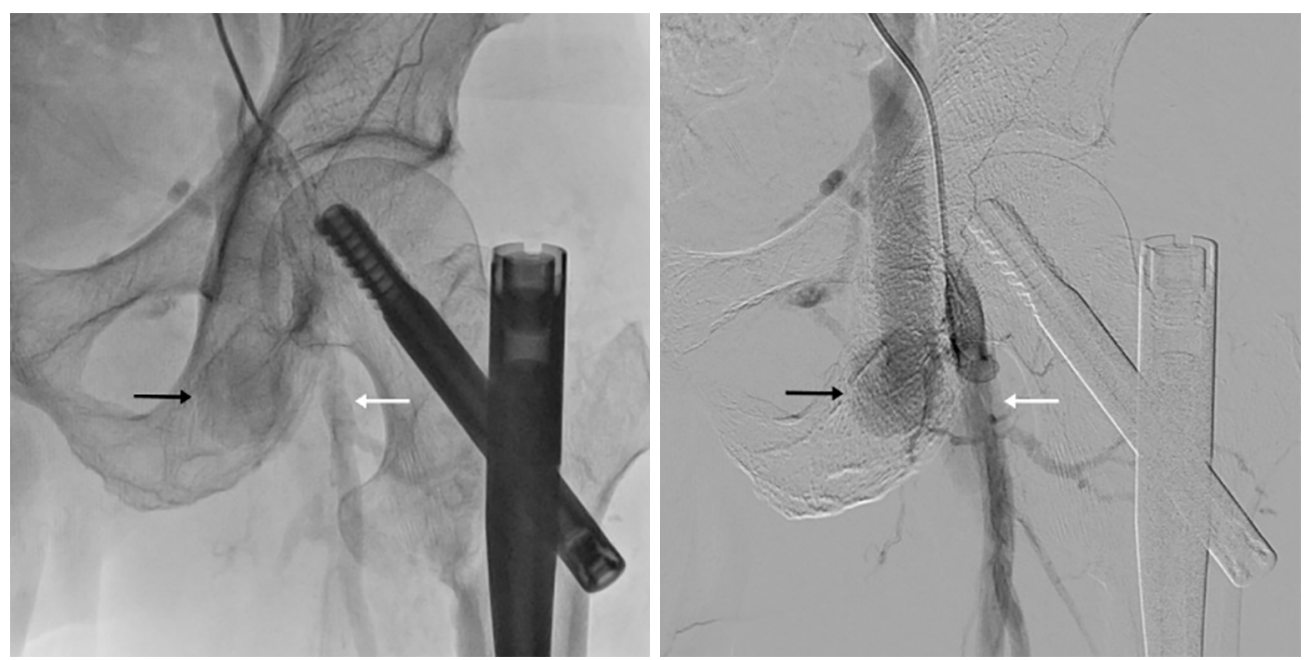

Figure 2 Preoperative angiography shows an abnormally enhanced vein at the arterial phase, resulting from the left femoral arteriovenous fistula associated with proximal femoral fracture. White arrows indicate the common femoral artery, and black arrows indicate the abnormally enhanced common femoral vein.

failed because the fistula tract was markedly ambiguous and the origin of the fistula tract was covered with the common, deep or superficial femoral artery. He was referred to the
Department of Thoracic and Cardiovascular Surgery for surgical management. The surgical approach was performed through a $4.0-\mathrm{cm}$ longitudinal skin incision in the left 

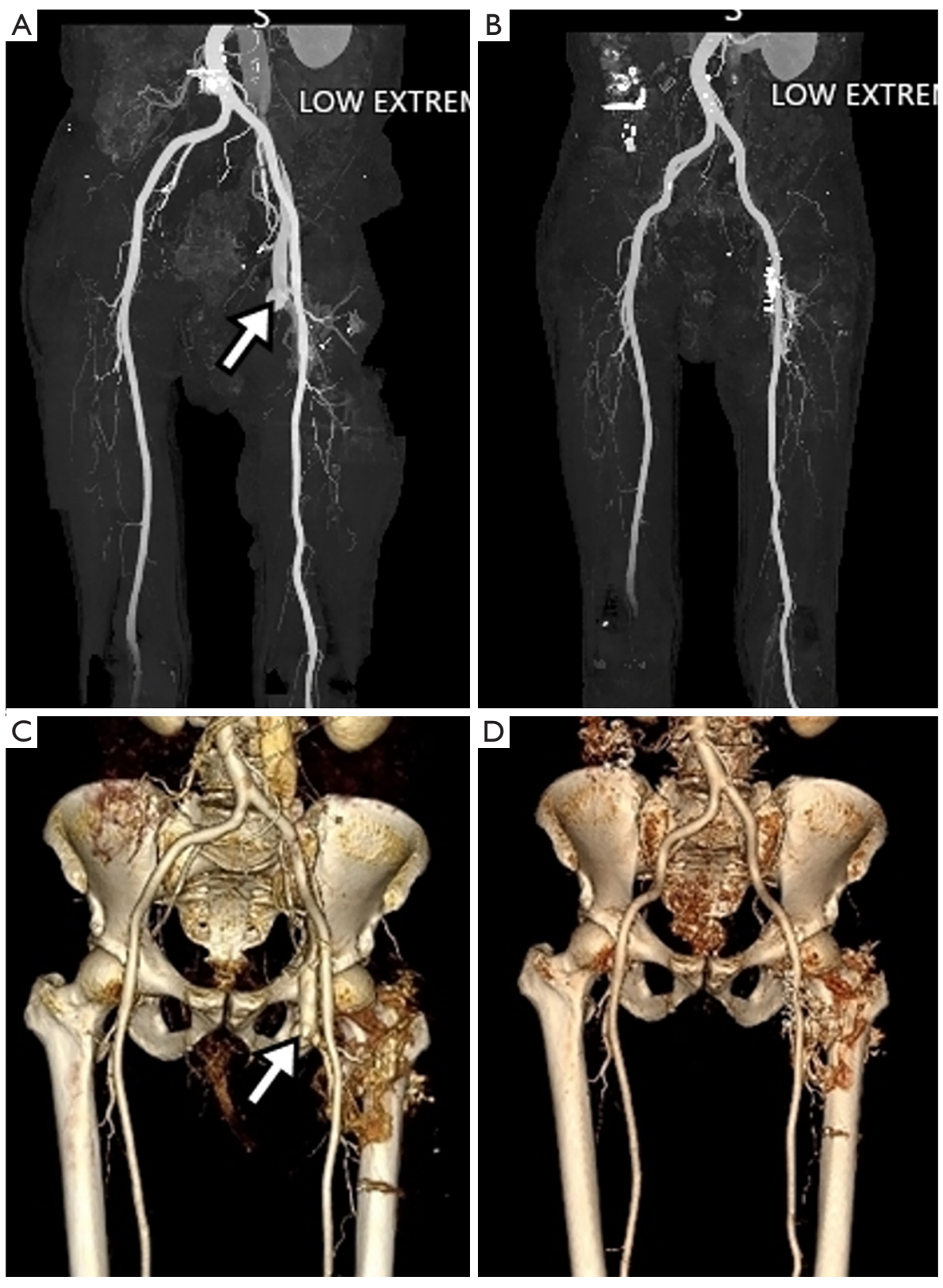

Figure 3 Angiographic 3D-reconstruction CT scans show enhanced veins at the arterial phase, resulting from a left femoral arteriovenous fistula associated with proximal femoral fracture (A,C, preoperative view) and a normalized vein at the arterial phase, after surgical correction of the left-sided femoral arteriovenous fistula (B,D, postoperative view).

inguinal area under general anesthesia. The intraoperative Nicoladoni-Branham sign was positive. The heart rate decreased from 80 to 55 beats/min after compression of the proximal common femoral artery, and returned to 80-90 approximately beats/min by compression release (8). The common, deep and superficial femoral arteries were separately dissected to detect the AVF. The AVF originated from the medial-inferior wall of each of the common, deep and superficial femoral arteries, which may have been attributed to iatrogenic, repetitive and multiple injuries to the femoral arteries and veins during surgery. It was fragile and had a thin sheet of soft tissue rather than vascular structure. It was ligated and resected, and then angioplasty was successfully performed. The patient recovered and was 

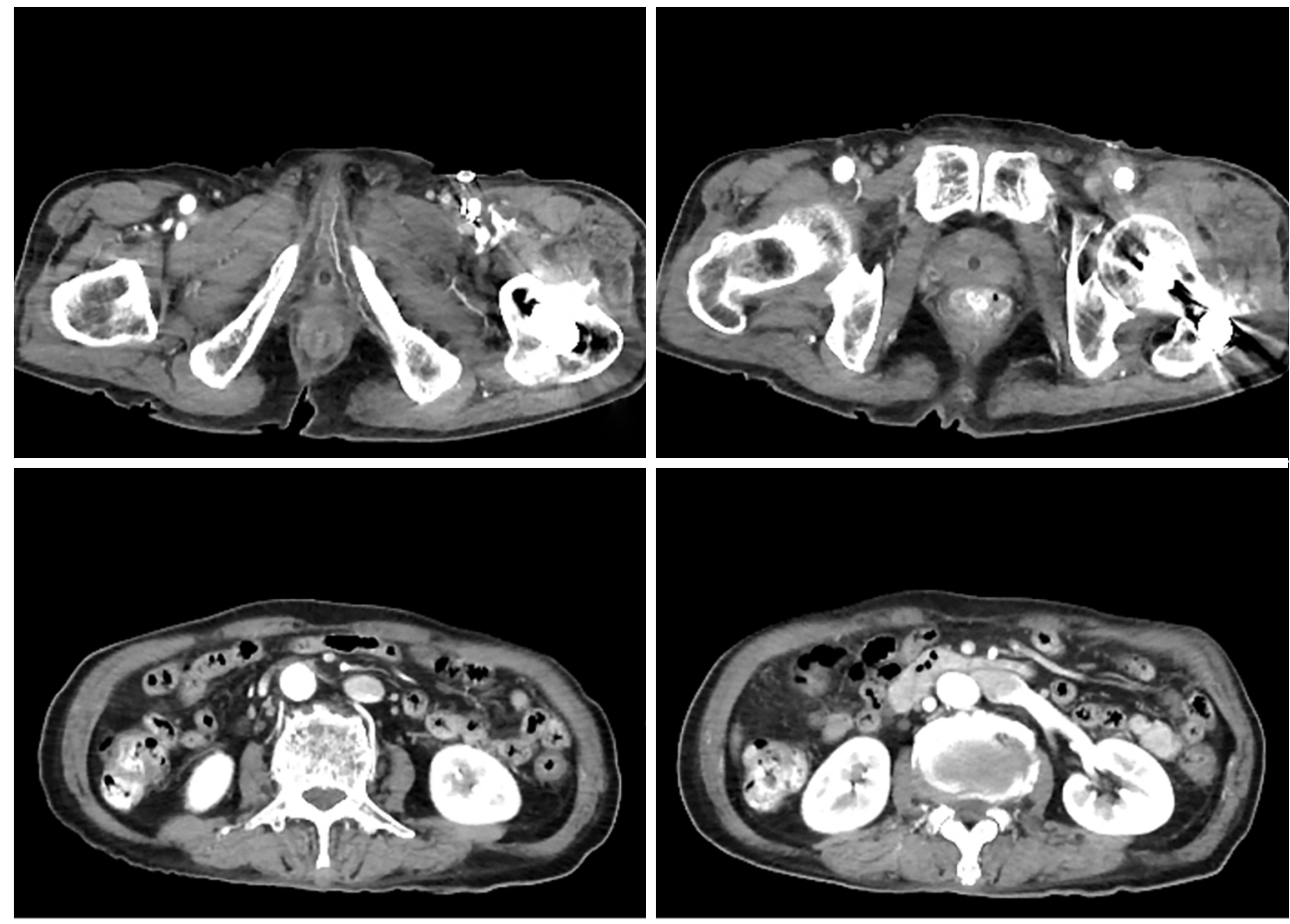

Figure 4 Postoperative transverse enhanced angiographic CT scans show normalized veins at the arterial phase, after successful surgical correction of the left femoral arteriovenous fistula. Compared to preoperative findings, the enhanced veins were normalized.

transferred to the Department of Rehabilitation Medicine. He was uneventfully discharged from the hospital (Figure 4).

\section{Results}

Forty-nine published studies were carefully analyzed, of which 30 were excluded and the remaining 19 were subjected to our analysis. Of these, only 7 met our inclusion criteria. Thus, eight studies, including seven previously published reports and one our case, were included in our qualitative assessment and systematic review (Figure 5). Clinical characteristics of the cases reported are summarized in Table 1 . For the 8 enrolled patients, mean age was $67.87 \pm 18.44$ years, and the male/female ratio was $1: 1$. Time to presentation was $743.55 \pm 946.94$ days after operation. The main symptoms were groin lump, pulsatile mass, and thigh swelling $(n=78)$, and there was a palpable thrill in 2 cases. Of the 8 patients, $6(75.0 \%)$ uneventfully underwent surgical treatments, including direct suture of injured vessels, angioplasty, patch angioplasty, embolization, branch ligation and coil embolization; $1(12.5 \%)$ was treated conservatively and died of multi-organ failure and hypovolemia; 1 (12.5\%) underwent angioplasty complicated by peroneal nerve impairment. in three cases. There was no description on the cause of vessel injury in 3 cases. There were 10 cases of fistula between the deep femoral artery and the deep femoral vein $(n=5)$, between the common femoral artery and the femoral vein $(n=3)$, between the superficial femoral artery and the common femoral vein $(\mathrm{n}=1)$, and between the popliteal artery and the popliteal vein $(n=1)$. The main causes of vessel injury were thought to be lesser trochanteric fracture fragment, retractor/drill bit and direct drilling; however, both of the exact mechanism and the cause of vessel injury were not available in the reports on three cases.

\section{Discussion}

Injuries to femoral vessels in PFF, including femoral neck and intertrochanteric, pertrochanteric and subtrochanteric fractures, are relatively rare, but critically fatal. PFF becoming exponentially prevalent, and it is at high risk of complication, morbidity and mortality. because it is frequently associated with serious traumas, advanced ages, poor underlying medical conditions, such as longstanding cancer, immunosuppressive condition, and 


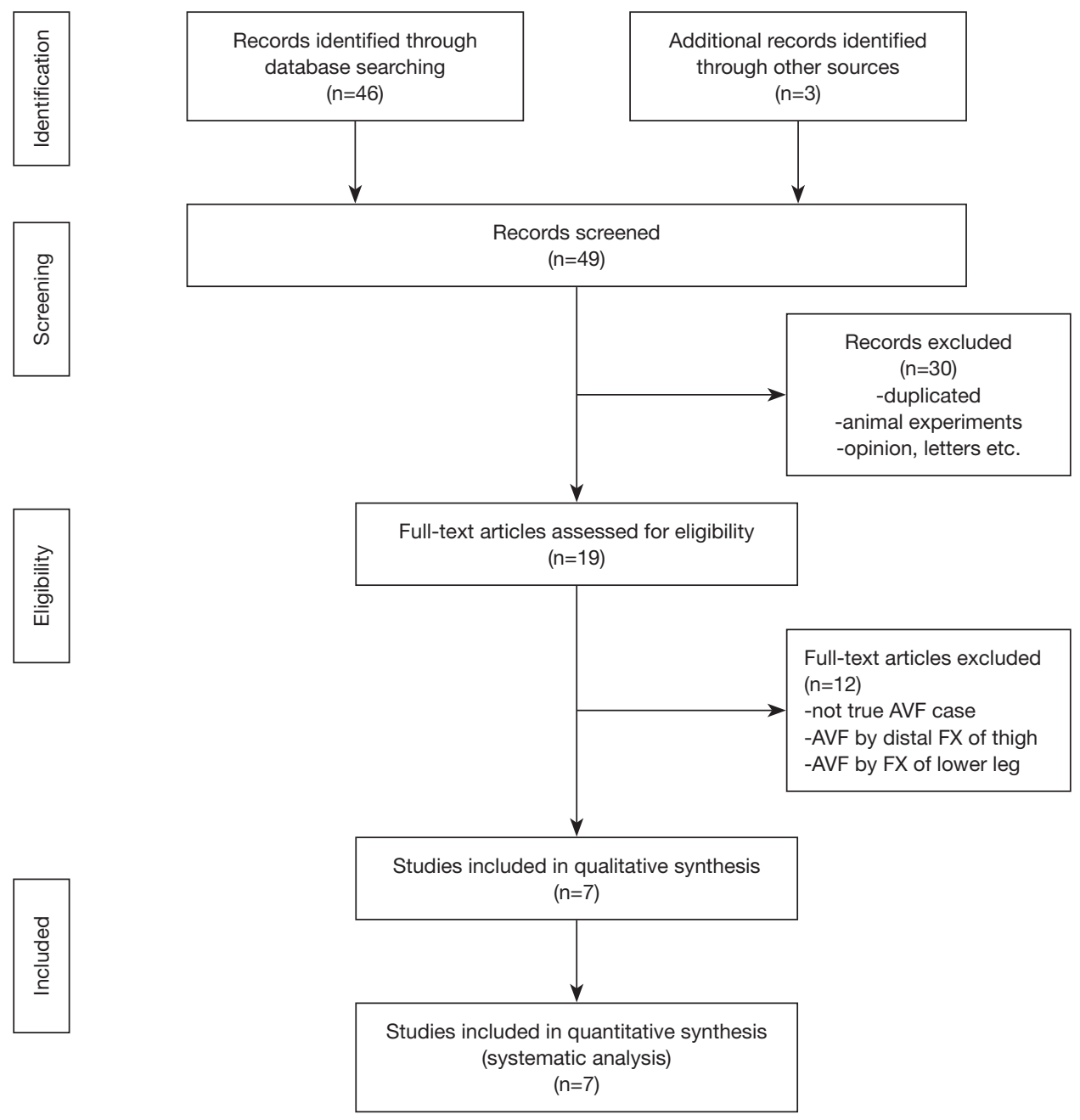

Figure 5 Flow diagram of the study selection process.

anticoagulant/antiplatelet treatment. There have been several case reports on vascular injuries to the external or internal iliac artery and the common, superficial or deep femoral artery following operation of fracture. In 1970, Müller (16) first pointed out the risk of vascular injury associated with hip surgery and recognized the importance of careful dissection of vascular structures during operation. Internal fixation and open reduction is an essential surgical treatment option. Unfortunately, some of the surgical procedures are related to vascular complications, especially in the deep, superficial or common femoral artery and its accompanying vein. Most of these vascular complications mainly involve the deep femoral artery, less often the superficial femoral artery, and rarely the common femoral artery. Mechanisms for femoral vessel injury have been proposed as follows: (I) a sharp and pointed bone fragment, especially from the lesser trochanter, causes a pressure/ stress injury to femoral vessels; (II) protruding cortical screws of the extramedullary implant cause a tip injury to femoral vessels during surgical manipulation; (III) direct injury by a mismatched distal locking screw, e.g., a Gamma intramedullary nail (Stryker, Portage, MI, USA), causes damage to the femoral vessels; (IV) inadvertent drilling or incorrect elevator placement induces vessel injuries; and (V) exothermic polymerization reactions to extruded bone cements, such as methyl methacrylate, occur. An excessive and repetitive pressure by a sharp bone fragment or a continuous contact with protruding tips of screws results in progressive erosion of femoral vessels and consequently develops vascular complications, such as vessel laceration, hemorrhage, thrombosis, embolism, intimal flap tear, false aneurysm, pseudoaneurysm and AVF. Vascular injuries 


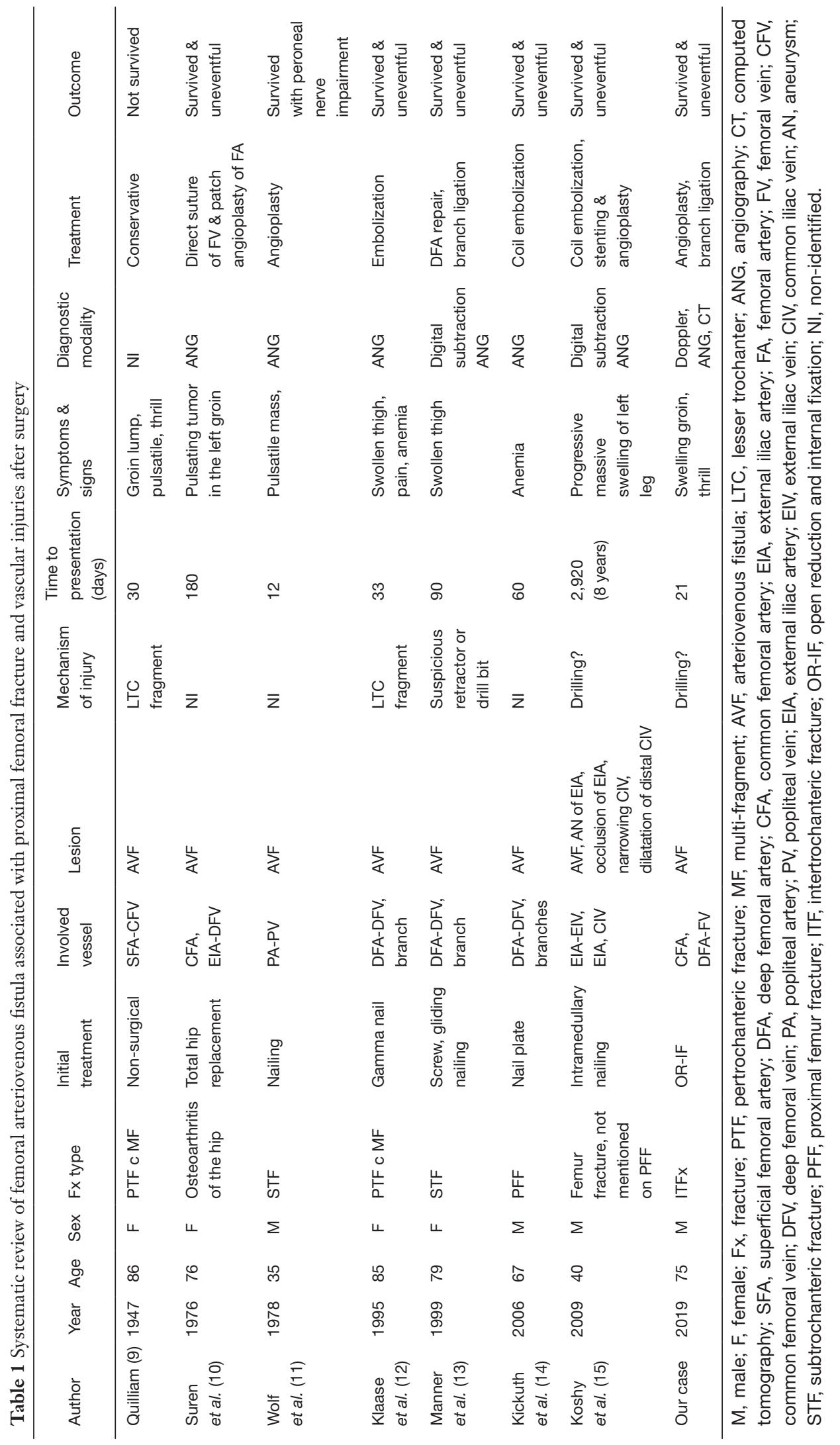


occurring during PFF surgery are potentially limb- and life-threatening. Some of the vascular problems associated with PFF can be clinically apparent several weeks or years after operation. The typical clinical features of vascular complications are an increased lower extremity swelling/pain, rapid swelling of the thigh with pulsation, more worsening pain, elevated skin temperature, venous dilatation in the lower extremity, increased diameter of the left lower extremity, and thrills/ bruits in the femoral region. In patients undergoing cardiac catheterization, most of the complications are presented as a gradually developing false pseudoaneurysm with swelling and pain; however, the incidence of femoral AVF is reported to be between 0 to $0.08 \%(17,18)$. Duplex Doppler ultrasonography, contrast enhanced computed tomography angiography, conventional angiography and magnetic resonance imaging are helpful in accurate diagnosis, evaluation and localization of injured vessels. Additionally, TTE is also helpful in evaluating significant ejection fraction and bi-atrial enlargement. Surgeons should rule out deep venous thrombosis, MayThurner's syndrome, primary/metastatic cancer, and soft tissue sarcoma. The successful treatment of femoral vascular complication mainly involving the deep femoral artery primarily depends on the status of the superficial femoral artery. In the case of an intact superficial femoral artery, trans-catheterization embolization or ligation of the deep femoral artery might be the best treatment option. In the case of an obstructed superficial femoral artery, the superficial or deep femoral artery should be surgically reconstructed, and direct injury to the superficial femoral artery should be repaired. Post-traumatic AVF should be obliterated as soon as possible, because untreated AVF frequently results in serious complications, including renin-angiotensin-aldosterone-mediated hypertension, high-output cardiac failure, and venous or arterial insufficiency. There are 2 treatment options for posttraumatic AVF: invasive surgical approaches, such as direct ligation and reconstruction of injured vessels and less invasive endovascular approaches, such as direct or transfemoral embolization with coils, closure with transluminal temporary occlusion of the pseudoaneurysm neck or balloon embolization, and direct thrombin injection. Surgical treatment is the gold standard for post-traumatic AVF, and endovascular procedures can be performed in limited cases.

Aust et al. (19) reported 5 cases of vascular complications following hip surgery, which can be explained by intraoperative injury. Buchholz et al. (20) report 2 cases of iliac pseudoaneurysm in 659 patients with infected arthroplasties. Bergqvist et al. (21) reported 4 cases of vascular complications after total hip replacement with the literature review of 25 cases of AVF, of which only 2 were associated with surgery of hip fractures, and which were same cases reported by Suren et al. and Tkaczuk. Suren et al. (10) also reported 2 patients with pelvic vessel complications after total hip replacement. One had a false aneurysm of the deep femoral artery; the other presented an AVF between the common femoral artery and its accompanying vein, in which scar tissue surrounding the AVF was severed, the femoral vein defect was managed by direct sutures, and the arterial lesion was repaired by patch angioplasty. Tkaczuk (22) reported a case of false aneurysm developing from the external iliac artery 8 months after total hip replacement in a 57-year-old woman. The arterial injury presenting as a false aneurysm developed by laceration of the external iliac artery and gradual erosion of a cement spicule which had entered the pelvis and pierced the external iliac artery. This case was not a true AVF, but a false aneurysm of the external iliac artery; therefore, the case of Tkaczuk was excluded from this review. There have been a few meta-analyses of iatrogenic arterial injuries associated with hip surgery. Shoenfeld et al. (23) reviewed 5 cases of vascular injuries after total hip replacement and additional 63 cases from the literature review to identify risk factors and clinical features. In the 68 patients, the left side was more common (66\%), the external iliac artery (36\%) was frequently involved, and common clinical findings were distal ischemia $(\mathrm{n}=31,46 \%)$, vessel laceration $(n=18,26 \%)$ and pseudoaneurysm $(n=17$, $25 \%)$. AVF ( $n=2,3 \%)$ less frequently developed than other vascular complications, which was consistent with the results reported by Bergqvist et al. (21). In 1991, Lazarides et al. (1) systematically reviewed a total of 69 articles and collected 126 cases of arterial trauma associated with hip joint surgery. In the 93 (74\%) of these cases arterial trauma occurred after total/subtotal hip replacement, 27 (21\%) were related to hip fracture correction, and 6 cases (5\%) complicated other hip surgical procedures. They also presented a detailed systematic overview of the literature along with their experiences in arterial injuries associated with hip replacement (group 1) and hip fracture correction (group 2), regarding locations, clinical features at presentation and mechanisms of injury. They summarized the differences in clinical characteristics between groups 1 and 2, which were summarized as follows: locations [external iliac artery (50\%) and common femoral artery (26\%) $v s$. deep femoral artery (77\%) and external iliac artery (7\%)], clinical features at presentation [ischemia (51\%) and false aneurysm $(30 \%)$ vs. false aneurysm (74\%) and hemorrhage 
(22\%)], and mechanisms of injury [cement spicule (31\%), retractor injury (28\%) and thermal injury (10\%) vs. screw injury (48\%), bone spike injury (20\%) and drilling (16\%)]. Of the 126 cases of iatrogenic arterial injuries associated with hip fracture, only 3 AVF cases occurred in group 1 and no AVF case in group 2. One was not a true AVF, but an abdominal-to-right hip joint fistula following repair of a large false aneurysm of the right external iliac artery in a 76-yearold woman. The pseudoaneurysm appeared as a complication 6 years after bilateral hip arthroplasty of PFF and the pseudo arteriovenous fistula from the abdomen to the right hip joint appeared as a complication of retroperitoneal abscess (24). Another was reported by Suren et al. (10), which was a true AVF associated with PFF and vascular injuries. The third was reported by Tkaczuk (22), which showed a false aneurysm of the external iliac artery and was excluded from this review. Barquet et al. (7) performed a systematic review on vascular injuries associated with internal fixation of PFF in adults, regarding incidence, etiology and outcome. They employed the PRISMA guidelines with no language restriction. They included a total of 160 articles with 182 cases of vascular injuries after internal fixation of PFF in the review. The injuries to extrapelvic vessels (the common, deep or superficial femoral artery and its accompanying vein) prevailed over those in intrapelvic vessels (the internal, external or common iliac artery and its accompanying vein. The types of injury were summarized as follows: compression, intimal flap tear, disruption of the intimal layer, thrombosis, laceration with hemorrhage, or progressive vessel erosion leading to a pseudoaneurysm or AVT. They found only 6 cases of arteriovenous aneurysm occurring in extrapelvic vessels (Table 1). They also showed that clinical and radiological findings in vascular injuries presenting during internal fixation of PFF were similar to those of previously published reports and that vascular injuries occurred either at the time of fracture and during or after internal fixation, early or late, weeks, months or even years after internal fixation. They concluded that the diagnostic and therapeutic modalities were noticeably diverse that the incidence of morbidity/ mortality was $18.06 \%$, and that the overall incidence of vascular injuries following internal fixation of $\mathrm{PFF}$ was $0.49 \%$.

Enhanced angiographic CT is essential for the evaluation of femoral AVF associated with PFF, which identifies homogeneous and high-density contrast-enhanced pathways and veins at the arterial phase. In patients with unexpected events, urgent angiography is essential for making an accurate diagnosis of fistula after lumber artery injury and for performing transcatheter arterial embolization. In a previous report, several mechanisms have been proposed for femoral AVF associated with PFF; however, the exact mechanism for this life/limb-threatening disease has not been fully elucidated. The morbidity and mortality can be lowered by early detection and proper treatment options, including transcatheter arterial embolization and surgical angioplasty. There is no consensus as to what is the best diagnostic or therapeutic modality. Surgeons must keep in mind that AVF requires early detection and quick treatment. The following factors cannot be underemphasized to prevent this complication: accurate diagnosis of the preoperative vascular status of the limb, detection of vascular insufficiency or ischemia, monitoring of the displacement of fracture fragments, careful and gentle reduction of the fracture fragments, careful placement of retractors, precise instrument handling, correct implant selection, and avoidance of trauma to the inner cortex of the acetabulum.

\section{Conclusions}

In conclusion, femoral AVF associated with operation of PFF can occur as an unusual event. Early detection and quick treatment not only prevent complications, such as cardiac failure and vascular insufficiency, but also avoid further surgical procedures.

\section{Acknowledgments}

Funding: None.

\section{Footnote}

Conflicts of Interest: The authors have no conflicts of interest to declare.

Ethical Statement: The authors are accountable for all aspects of the work in ensuring that questions related to the accuracy or integrity of any part of the work are appropriately investigated and resolved. The present study was approved by the Institutional Ethics Committee/ Review Board of Konkuk University Chungju Hospital, which waived the requirement for informed patient consent due to its retrospective case reporting nature (IRB approval No. KUCH 2019-10-032).

Open Access Statement: This is an Open Access article distributed in accordance with the Creative Commons Attribution-NonCommercial-NoDerivs 4.0 International 
License (CC BY-NC-ND 4.0), which permits the noncommercial replication and distribution of the article with the strict proviso that no changes or edits are made and the original work is properly cited (including links to both the formal publication through the relevant DOI and the license). See: https://creativecommons.org/licenses/by-nc-nd/4.0/.

\section{References}

1. Lazarides MK, Arvanitis DP, Dayantas JN. Iatrogenic arterial trauma associated with hip joint surgery: an overview. Eur J Vasc Surg 1991;5:549-56.

2. Karanikas I, Lazarides M, Arvanitis D, et al. Jatrogenic arterial trauma associated with hip fracture surgery. Acta Chir Belg 1993;93:284-6.

3. Osagie L, Gallivan S, Pearse Y. Profunda femoris injury following lesser trochanter displacement: complications following intramedullary femoral nailing. Injury 2015;46:411-3.

4. Bartonícek J. Injuries to femoral vessels in fractures of the hip. Rozhl Chir 2009;88:203-5.

5. Waddell JP. editor. Complications of trochanteric fractures. In: Fractures of the proximal femur improving outcome. Philadelphia, PA: Saunders; 2011:151-84.

6. Moher D, Liberati A, Tetzlaff J, et al. Preferred reporting items for systematic reviews and meta-analyses: the PRISMA statement. BMJ 2009;339:b2535.

7. Barquet A, Gelink A, Giannoudis PV. Proximal femoral fractures and vascular injuries in adults: Incidence, aetiology and outcomes. Injury 2015;46:2297-313.

8. Burchell HB. Observations on bradycardia produced by occlusion of an artery proximal to an arteriovenous fistula (Nicoladoni-Branham sign). Med Clin North Am 1958;42:1029-35.

9. Quilliam TA. Arteriovenous aneurysm as a complication of intertrochanteric fracture of the femur. Br J Surg 1947;34:327.

10. Suren EG, Mellmann JR, Leitz KH. Vascular complications following alloplastic hip replacement. Arch Orthop Unfallchir 1976;85:217-24.

11. Wolf JW Jr, Tegtmeyer CJ, Whitehill R, et al. Traumatic arteriovenous fistula of the popliteal artery associated with a closed subtrochanteric fracture: report of a case. Clin Orthop Relat Res 1978;133:227-9.

12. Klaase JM, van Walsum AD, Vierhout PA, et al. Letsel van de arteria profunda femoris bij een pertrochantere femurfractuur. Ned Tijdschr Traum 1995;2:162-5.

13. Manner M, Rösch B, Roy K. Vascular injuries complicating osteosynthesis in proximal femur fractures. Unfallchirurg 1999;102:227-31.

14. Kickuth R, Anderson S, Kocovic L, et al. Endovascular treatment of arterial injury as an uncommon complication after orthopedic surgery. J Vasc Interv Radiol 2006;17:791-9.

15. Koshy CG, Keshava SN, Surendrababu NR, et al. Endovascular management of posttraumatic arteriovenous fistulae. Cardiovasc Intervent Radiol 2009;32:1042-52.

16. Müller ME. Total hip prostheses. Clin Orthop Relat Res 1970;72:46-68.

17. Bitargil M, Başbuğ HS, Göçer H, Günerhan Y, Karakurt A. Coronary angiography our approaches to vascular complications. Turkish J Vasc Surg 2014;23:164-8.

18. Kelm M, Perings SM, Jax T, et al. Incidence and clinical outcome of iatrogenic femoral arteriovenous fistulas: implications for risk stratification and treatment. J Am Coll Cardiol 2002;40:291-7.

19. Aust JC, Bredenberg CE, Murray DG. Mechanisms of arterial injuries associated with total hip replacement. Arch Surg 1981;116:345-9.

20. Buchholz HW, Elson RA, Engelbrecht E, et al. Management of deep infection of total hip replacement. J Bone Joint Surg Br 1981;63-B:342-53.

21. Bergqvist D, Carlsson AS, Ericsson BF. Vascular complications after total hip arthroplasty. Acta Orthop Scand 1983;54:157-63.

22. Tkaczuk H. False aneurysm of the external iliac artery following hip endoprosthesis. Acta Orthop Scand 1976;47:317-9.

23. Shoenfeld NA, Stuchin SA, Pearl R, et al. The management of vascular injuries associated with total hip arthroplasty. J Vasc Surg 1990;11:549-55.

24. Korovesis P, Siablis D, Salonikidis P, et al. Abdominal-hip joint fistula. Complicated revision of total hip arthroplasty for false aneurysm of external iliac artery A case report. Clin Orthop Relat Res 1988;231:71-5.

Cite this article as: Kim JS, Lee SA, Chee HK, Hwang JJ, Kim HY, Kim JY, Choi SM, Kim YH, Lee WS. Femoral arteriovenous fistula associated with surgery of proximal femoral fracture: a systematic review of the literature and case presentation. Ann Transl Med 2020;8(6):291. doi: 10.21037/ atm.2020.03.08 